\title{
¿DÓNDE ESTÁN Y QUÉ HACEN LAS MUJERES DENTRO DE LA ADMINISTRACIÓN PÚBLICA? EL CASO DEL SECTOR PÚBLICO ARGENTINO
}

\author{
Mariana Chudnovsky \\ Centro de Investigación y Docencia Económicas, México \\ mariana.chudnovsky@cide.edu
}

\section{RESUMEN}

Argentina es uno de los países con mayor igualdad de género, sobre todo, debido al aumento en la paridad de género en el Poder Legislativo. Sin embargo, esto no se traduce al Poder Ejecutivo: el país no solo muestra una baja posición, sino también una disminución de mujeres en posiciones ministeriales. Este artículo describe la situación de las mujeres en todos los niveles de la rama ejecutiva con el objetivo de identificar dónde están las mujeres, qué están haciendo y cuánto ganan por su trabajo. Los hallazgos más significativos son que los hombres ocupan la mayoría de las posiciones del gabinete político y de las posiciones gerenciales. En el nivel burocrático, se observa que las mujeres tienen más grados universitarios que los hombres, se concentran en los niveles más bajos de la jerarquía (el escalafón vigente) y ganan menos que los hombres en la misma posición.

Palabras clave: Género, Burocracia, Servicio civil, Argentina. 


\title{
WHERE ARE WOMEN IN THE PUBLIC ADMINISTRATION AND WHAT DO THEY DO? THE CASE OF ARGENTINA'S PUBLIC SECTOR
}

\begin{abstract}
Argentina is one of the countries with the highest level of gender equality due, above all, to the increase in gender parity in the legislature. However, this is not the case in the executive where their participation is low and the number of women in ministerial positions has decreased. This article describes the situation of women at all levels of the executive branch, seeking to identify where they are, what they are doing and how much they earn from their work. The most significant findings are that men occupy the majority of positions in the political cabinet and in managerial positions. At the bureaucratic level, women are found to have more university degrees than men, are concentrated in the lowest levels of the hierarchy (the current scale of seniority) and earn less than men in the same position.
\end{abstract}

Keywords: Gender, Bureaucracy, Civil service, Argentina. 


\section{INTRODUCCIÓN}

En América Latina, durante los años noventa, diferentes gobiernos hicieron importantes progresos en el establecimiento de cuotas de género en la rama legislativa y obtuvieron resultados positivos (Rodríguez Gustá y Caminotti 2010, Marx, Borner y Caminotti 2007). Sin embargo, esos grandes logros en el Poder Legislativo no fueron equivalentes en el Poder Ejecutivo. Este trabajo busca documentar esa diferencia de estrategias en ambos poderes. Para hacerlo, toma en cuenta que la Plataforma de la Cuarta Conferencia Mundial sobre Mujeres en Beijing (1995) estableció la necesidad de combinar dos guías estratégicas: por una parte, acciones afirmativas que favorezcan el acceso de las mujeres a las posiciones electivas $y$, por otra parte, el enfoque de integración o transversalización de género en el Estado, con el objetivo de promover igualdad a través de un enfoque de género en todos los sistemas y estructuras (Daly 2005, Connell 2006). Este artículo se centra en el caso argentino, ya que ha sido reconocido como un país pionero en las acciones afirmativas en el Poder Legislativo. Con la promulgación de la Ley de Cuotas de Mujeres en 1991, fue el primer país en usar la estrategia para incorporar a las mujeres en posiciones políticas legislativas electivas con el objetivo de alcanzar la paridad legislativa. La cantidad de mujeres que ocupan asientos en el Congreso Nacional también ha crecido en años recientes, principalmente debido a la Ley de Cuotas de 1991 (Rodríguez Gustá y Caminotti 2010). Sin embargo, este progreso en la rama legislativa no se reflejó en un avance equivalente en el Poder Ejecutivo, donde el nivel de participación femenina muestra un crecimiento más tímido e inestable (CIPPEC 2017).

América Latina y el Caribe puntúan 29\% en el total de brecha de género, y se ubican en el medio del rango del índice (World Economic Forum 2018). Argentina (27\%) es uno de los países con mayor igualdad de género en la región y muestra un incremento en el subíndice de empoderamiento político, el cual se explica, justamente, por el aumento en la paridad de género en la proporción de mujeres legisladoras. Sin embargo, la evidencia muestra que esto no se traduce al Poder Ejecutivo: el país no solamente muestra una baja posición considerando el Ejecutivo, sino también una disminución de mujeres en posiciones ministeriales (World Economic Forum 2017: 20). De acuerdo con el Índice Global de Brechas de Género (en dónde 0 implica la total disparidad de género y 1 la total paridad) (World Economic Forum 2018), Argentina muestra un incremento en las cuotas de legisladoras femeninas $(0,6)$, pero, en cambio, presenta una disminución de mujeres en posiciones ministeriales $(0,2)$. Este avance dispar llama la atención ya que no es un problema de descuido generalizado 
del tema por parte de las diferentes administraciones políticas, dado que sí le han dado importancia en el área legislativa.

Para dar un primer paso en abordar este asunto crucial, en este artículo se realiza un estudio de caso en profundidad para describir la situación de las mujeres en todos los niveles de la rama ejecutiva. Se busca identificar dónde están las mujeres en el Poder Ejecutivo, qué están haciendo y cuánto ganan por su trabajo. Para ello, se examina la presencia de mujeres en el alto gobierno (políticos), alta gerencia pública (posiciones ejecutivas) y el nivel administrativo (burocracia y contratos específicos de esemismo nivel jerárquico) desde 2001 hasta 2017. También examinamos brechas salariales, posiciones jerárquicas y nivel educativo entre hombres y mujeres.

El artículo se organiza de la siguiente manera: la siguiente sección revisa la literatura relevante; la tercera presenta el diseño de investigación, el universo del estudio y las fuentes de información; el cuarto apartado describe el funcionamiento del empleo público en el sector público federal de Argentina; la quinta sección examina la presencia de mujeres en niveles de la pirámide del empleo público; finalmente, presentamos conclusiones y discusiones de posibles soluciones de políticas.

\section{GÉNERO Y BUROCRACIA}

A pesar de que muchos debates sobre burocracias representativas existieron por un largo tiempo en los países centrales (Atkins y Wilkins 2013, Bradbury y Kellough 2011, Keiser et al. 2002, Kingsley 1944, Lewis y Pitts 2011, Meier y Nicholson-Crotty 2006, Meier y Nigro 1976, Meier y Stewart 1992), en Latinoamérica ese programa de investigación no ha tenido tanto impacto. En términos generales, podemos definir burocracias pasivas como aquellas burocracias que comparten el mismo origen demográfico que la población general y burocracias activas como aquellas que, además, producen resultados de políticas que benefician a esos ciudadanos que están pasivamente representados (Meier 1993).

Desde la década de 1970, existe evidencia acerca de la idea de que la representación burocrática importa. Las burocracias debieran representar y reflejar a la población a la que sirven (Bowling et al. 2006). Asimismo, existen estudios sobre burocracia representativa que señalan que la composición demográfica del sector público influye tanto en la naturaleza de los gobiernos como en los resultados de las políticas (Kranz 1976; Meier y Nigro 1976). Por ejemplo, Dolan (2004: 300) señala que "un sector público diverso es importante no solamente por razones simbólicas, sino porque se espera que las decisiones del gobierno sean más responsivas 
al público cuando la fuerza de trabajo es más diversa”. En suma, la representación debe incluir representación activa y pasiva.

También existe evidencia del impacto de la representación burocrática en términos de género (Bowling et al. 2006, Fernández, Malatesta y Smith 2013, Meier y Nicholson-Crotty 2006, Park 2013, Riccucci y Saidel 1997, Selden 2006, Wilkins 2007). Sin embargo, las mujeres deben ser capaces de obtener posiciones de liderazgo, además de tener presencia y representación pasiva. Si ellas tienen mucha menos influencia que los hombres en la toma de decisiones políticas, la cadena de representación se rompe.

De hecho, existen diferencias significativas en el perfil de los cargos directivos que ocupan y que, además, esta brecha de género predice la brecha salarial (Alkadry y Tower 2014). De hecho, Kerr, Miller y Reid (2002) encuentra un nivel significativo de segregación ocupacional horizontal entre administradores en agencias estatales en Estados Unidos y Lewis y Soo Oh (2009) encuentran que la segregación ocupacional explica parte de la brecha salarial de género.

La importancia del acceso femenino a posiciones gerenciales y ejecutivas dentro de la administración pública ha ganado atención alrededor del mundo (Bowling et al. 2006). Existe evidencia del impacto positivo de la diversidad de género (Riccucci y Saidel 1997, Alkadry y Tower 2014) y sobre su influencia en los niveles más altos sobre el desempeño financiero de las organizaciones públicas (Opstrup y Villadsen 2014) y la disminución de los niveles de corrupción (Jin 2017). Sin embargo, solo el 34\% de las posiciones de liderazgo del servicio público son sostenidas por mujeres en el mundo (World Economic Forum 2018) y hay escasa evidencia empírica que considere lo que sucede en América Latina.

En América Latina, la escasa presencia de iniciativas orientadas al desarrollo de mecanismos de burocracias representativas y el poco debate sobre su rol en la representación es notorio. Un claro indicador de ello es que no existe ningún tipo de cuotas o políticas de género (u otras minorías) en las diferentes legislaciones de servicio civil en la región (Cortázar et al. 2014).

\section{IGUALDAD DE GÉNERO: SEGREGACIÓN VERTICAL Y HORIZONTAL}

Existe evidencia que muestra que las mujeres ganan menos que los hombres que realizan sus mismas tareas (D'Agostino 2015, Alkadry et al. 2019, Bishu y Alkadry 2017, Choi 2018). El género inncide en el pago, incluso 
controlando por años de experiencia, negociaciones colectivas, educación y otras variables de capital humano (Alkadry y Tower 2014). En general, las mujeres enfrentan más dificultades que los hombres para acceder a mejores empleos pagados, los cuales implican mayores perspectivas profesionales y mayor protección social (García 2003, Rodríguez 2008). Asimismo, las mujeres han asumido roles subordinados en las organizaciones (CáceresRodríguez 2013). Esta segregación vertical ha sido estudiada bajo el nombre de techo de cristal.

La segregación vertical implica que las mujeres ingresan a las organizaciones en niveles jerárquicos más bajos que los hombres y luego enfrentan mayores dificultades para ascender a los puestos directivos. Por ejemplo, Gaete y Álvarez (2020) muestran que en el servicio vivil chileno, solo $29 \%$ de los concursos realizados entre 2010 y 2017, fueron adjudicados a mujeres. En cambio, los hombres obtienen posiciones más altas en gobiernos federales (Dolan 2004) y locales (Kerr, Miller y Reid 2002), así como también en agencias públicas particulares (Bishu y Headley 2020, Riccucci y Saidel 1997), empresas privadas (Camarena y Saavedra 2018) y en la academia (Gaete, Álvarez y Ramírez 2019). Además, existe evidencia de que, incluso después de alcanzar un puesto de liderazgo, las mujeres supervisan a menos subordinados y tienen menos responsabilidad financiera en comparación con los hombres (Alkadry et al. 2019). Además del techo de cristal, existe la segregación horizontal: las mujeres tienden a estar sobrerrepresentadas en ciertas ocupaciones como servicios sociales, educación y cuidados, mientras que tienden a estar subrrepresentadas en ocupaciones tradicionalmente, dominadas por hombres como ingeniería y finanzas (Alkadry et al.2019, Alkadry y Tower 2014).

A pesar de que en las últimas décadas las mujeres han avanzado en posiciones de liderazgo, aún enfrentan serios obstáculos. Tal como fue descrito por Sabharwal (2013: 675), cuando las mujeres logran ascender, "ellas a menudo son puestas en posiciones precarias que las empujan al fracaso-un fenómeno recientemente conocido como 'acantilado de cristal'”. De igual manera, el liderazgo a menudo se relaciona con características masculinas que contradicen las imágenes femeninas de cuidado asociadas con las mujeres (Stivers 2002) y este estereotipo puede afectar la forma en que las mujeres y los hombres son tratados en las posiciones de liderazgo. En particular, la incongruencia entre lo que significa ser mujer y lo que se valora en un rol de liderazgo puede conducir a evaluaciones menos favorables del comportamiento real de las mujeres líderes (Ryan y Haslam 2007). Por lo tanto, las mujeres tienen más probabilidades de fracasar cuando se elevan en la organización. 


\section{EL CONTRASTE ENTRE EL PODER LEGISLATIVO Y EL EJECUTIVO}

En América Latina, surgieron diversas acciones mejorar la situación de las mujeres. En general, se centraron en políticas de acción afirmativa en los poderes legislativos. En la década de 1990 doce países de la región adoptaron cuotas electorales para la participación femenina en los poderes legislativos (Rodríguez Gustá y Caminotti 2010). En su perspectiva más clásica, las acciones afirmativas son procedimientos que buscan garantizar la representación de grupos discriminados. Algunos ejemplos clásicos son las minorías de género (mujeres), raciales, étnicas, etarias, entre otras (Ridgeway y Correll 2004).

En 1990, la presencia de mujeres en las cámaras bajas (o únicas) de los países de la región no excedieron, en promedio, el 9\% de los asientos (Barreiro et al. 2004). En ese contexto, la década de 1990, fue escenario de un conjunto de demandas y negociaciones nacionales que guiarían a la adopción de cuotas de género para las candidaturas legislativas de muchos países de la región (Marx, Borner y Caminotti 2007).

La convención sobre la Eliminación de Todas las Formas de Discriminación contra la Mujer (CEDAW) es un tratado internacional adoptado en 1979 por la Asamblea General de las Naciones Unidas y en 1990 ya había sido ratificada por todos los Estados de América Latina. Desde entonces, y sobre todo, después de la Conferencia de Beijing (1995), la adopción cuotas electorales para la participación femenina se popularizó en la región. En general, las leyes de cuota establecen porcentajes mínimos de mujeres candidatas (alrededor de 30\%) o también porcentajes mínimos y máximos de candidatos por género. El uso de cuotas proliferó en el mundo de diferentes maneras. Por ejemplo, en Europa Occidental las cuotas fueron promovidas por el partido, mientras que en Latinoamérica se fomentaron las cuotas legalmente (Marx, Borner y Caminotti 2007).

Las cuotas están basadas en una distinción entre una noción de igualdad de oportunidades (igualdad formal) e igualdad de resultados (igualdad real) (Rodríguez 2008). Desde esta perspectiva, su principal objetivo es contrarrestar los privilegios que los hombres han tenido en la hisotria, de acuerdo a la condición dominante del hombre en la vida pública (Bacchi 2006). Esto es por qué los derechos políticos de las mujeres son, desde esta perspectiva, derechos humanos y ciudadanos.

El caso argentino no estuvo fuera de esta tendencia. De hecho, fue icónico en términos de avances en cuotas legales. En 1991, con la promulgación 
de la Ley de Cuotas de Mujeres, el país se convirtió en el primer país del mundo en adoptar un mínimo de cuotas para candidatas legislativas mujeres. Se reformó la legislación electoral y todos los partidos políticos debieron incorporar a mujeres en sus listas electorales "en un mínimo de $30 \%$ de los candidatos a posiciones electivas y en proporciones con la posibilidad de ser electas" (Marx, Borner y Caminotti 2007).

En 2017, el porcentaje de cuotas fue modificado, con la promulgación de una nueva ley, la cual establece un sistema de paridad de género (50\%) en el campo de la representación política. Esto es tano para las listas de candidatos por el Congreso Nacional (diputados y senadores) como para el Parlamento Mercosur. En la Cámara de Diputados, las mujeres pasaron de representar 6\% en 1991 a 38,5\% en 2018. En el Senado, pasaron de representar $8 \%$ a $41,6 \%$. Queda claro que las iniciativas de cuotas legales fueron cumplidas.

\section{DISEÑO DE INVESTIGACIÓN, MÉTODOS YTÉCNICAS}

El diseño de investigación consiste en un estudio de caso descriptivo para realizar un primer acercamiento a un tema con aún escasa evidencia empírica acerca de qué sucede con las mujeres en las administraciones públicas de la región. Asimismo, se centra en el nivel federal de gobierno debido a la complejidad de obtener información de la administración pública en el nivel subnacional de gobierno. Las fuentes de información utilizadas para este estudio se basan en información obtenida en el Ministerio de Hacienda de la Argentina y en publicaciones de su página web institucional.

Se considera a la cantidad de personal teniendo en cuenta aquel que, efectivamente, ocupa un cargo que se encuentra financiado por el Presupuesto General de la Administración Pública Nacional y en la estructura correspondiente a cada nivel institucional. Para mostrar la participación de las mujeres en cada nivel analizado, se indagó el dato de tal forma de poder desagregarlo cualitativamente, a efectos de poder medir el nivel educativo de cada agente, el rango etario y el salario promedio.

El universo de estudio de este trabajo es el Poder Ejecutivo Nacional (PEN), que engloba a la Presidencia de la Nación, la Jefatura de Gabinete de Ministros y los Ministerios Nacionales. Dentro de este universo, se examinan a los empleados civiles del PEN, puesto que es el órgano encargado de administrar, aplicar y ejecutar las leyes y las políticas públicas. El principal escalafón que rige el empleo público es el Sistema Nacional de Empleo Público (SINEP) en el marco de la Ley Marco de Regulación del Empleo Público No 25.164 (en adelante, Ley Marco). El personal que 
se desempeña en el PEN puede revestir en el régimen de estabilidad (lo que se denomina planta permanente), en el régimen de contrataciones (los contratos por tiempo determinado) o como alto gobierno: las autoridades superiores, los funcionarios con roles políticos nombrados por fuera del escalafón vigente (extraescalafonarios) o asesores de gabinete.

La normativa vigente dispone que a la planta permanente se accede mediante un concurso, que es el mecanismo legal que permite medir la idoneidad y la capacidad de la persona. Dicho cargo debe estar financiado en la Ley de Presupuesto aprobada por el Congreso de la Nación. La estabilidad en el cargo del empleado público está garantizada por la Constitución Nacional (artículo 14 bis) y por el artículo $8^{\circ}$ de la Ley Marco. Asimismo, la normativa establece que la designación de personal sin la mediación de los sistemas de selección previstos no reviste en ningún caso carácter de permanente, ni genera derecho a la estabilidad.

Se entiende por alto gobierno a los máximos niveles políticos de conducción, como el Presidente, Vicepresidente, Jefe de Gabinete, ministros, viceministros, secretarios y subsecretarios o posiciones de esa jerarquía (Chudnovsky y Cafarelli 2018). En el caso argentino, también lo conforman el personal extraescalafonario y los asesores de gabinete. Estos se designan mediante decreto presidencial y continúan hasta que finalice el mandato o hasta que el presidente decida removerlos. El personal extraescalafonario se compone por las máximas autoridades de los organismos descentralizados e instituciones de la seguridad social, son nombrados por el PEN y su cantidad depende del poder político, ya que ellos son los que están autorizados a crear o suprimir estos cargos. Los asesores de gabinete son elegidos por las autoridades políticas para realizar tareas que se limitan exclusivamente al asesoramiento y asistencia administrativa a dichas autoridades, excluyendo las tareas correspondientes a los niveles de conducción y jefatura de la planta estatal.

Se considera a la Alta Dirección Pública (ADP) como a aquella conformada por directores nacionales, generales y simples, coordinadores o jefes de área designados por un período de cinco años. Según la Ley Marco a estos puestos se accede mediante un sistema de selección que permita medir la idoneidad de la persona para ocupar el puesto, que suele ser el concurso. Aunque deberían ingresar por este sistema, en muchas oportunidades lo hacen por decreto de designación transitoria debido a una situación de excepción a la Ley Marco de Empleo Público vigente. Del total de funcionarios que conforman la ADP, el 85\% se encuentra designado mediante excepción a la norma por un plazo de 180 días. Solo un $15 \%$ accedió mediante un concurso, sin embargo, el cargo directivo 
lo tienen vencido ya que en los últimos años casi no hubo llamados a concurso (Chudnovsky y Cafarelli 2018).

El nivel administrativo alude al servicio civil propiamente dicho, sin incluir cargos directivos ni políticos. Dentro de este universo, examinamos a los empleados civiles del escalafón SINEP del PEN, puesto que es el órgano encargado de administrar, aplicar y ejecutar las leyes y las políticas públicas. A la fecha bajo análisis, el SINEP es el escalafón civil con ocupación mayoritaria, que tiene la particularidad de ser transversal a los organismos que componen este poder (Dirección de Evaluación Presupuestaria Salarial 2017). A este escalafón se accede mediante un concurso (planta permanente) o mediante un contrato por tiempo determinado. Ambos sistemas de acceso se encuentran regulados en la Ley Marco.

\section{PRESENCIA DE MUJERES EN LA TOMA DECISIONES DENTRO DE LOS PODERES EJECUTIVOS: GABINETE DEL PRESIDENTE Y ADMINISTRACIÓN PÚBLICA}

La Tabla 1 sintetiza la presencia de las mujeres en el alto gobierno (los políticos), la alta dirección pública (los cargos directivos) y el nivel administrativo (compuesto por el servicio civil de carrera y los contratos asimilables a ese nivel administrativo) para el año 2017.

Tabla 1: Participación porcentual del personal civil por género en el PEN, 2017

Femenino Masculino

Personal Civil del PEN

Alto gobierno

Alta Dirección Publica

Nivel administrativo
$41 \%$

$25 \%$

$41 \%$

$53 \%$
$59 \%$

$75 \%$

$59 \%$

$47 \%$

Fuente: Elaboración propia con base en datos publicados el Ministerio de Hacienda.

A continuación, presentamos los resultados del análisis para cada uno de estos segmentos. 


\section{EL ALTO GOBIERNO}

Al examinar los nombramientos políticos por género dentro del alto gobierno, la cantidad de mujeres es escasa, aunque muestra una tendencia creciente. En el año 2001 las mujeres representaban el 13\% y en el 2017 conformaban el $25 \%$ de los cargos de alto gobierno. Además de su baja presencia en puestos de decisión, se observa un desplazamiento de las mujeres del gabinete del presidente a ciertas áreas ocupacionales específicas, habitualmente consideradas femeninas o de menor prestigio (CIPPEC 2017).

Los ministerios de Defensa, Hacienda, Ciencia, Tecnología e Innovación Productiva y Finanzas no tenían, en 2016, ningún cargo político (ministra, secretarias o subsecretarias) ejercido por una mujer. En cambio, entre 2011 y 2015 estos mismos ministerios contaban con un 11\%, 27\% y $30 \%$, respectivamente, es decir, que no hubo mejoras. En esos años, los ministerios con mayor presencia de mujeres eran los de Desarrollo Social (43\% en 2016; 50\% entre 2011-2015), Salud (40\% en 2016; $20 \%$ entre 2011-2015) y Educación (36\% en 2016; 29\% entre 2011-2015).

El Gráfico 1 muestra la evolución porcentual de la cantidad de puestos políticos ocupados por mujeres y hombres durante los ańos 2001 a 2017:

Gráfico 1: Alto Gobierno por género, 2001-2017

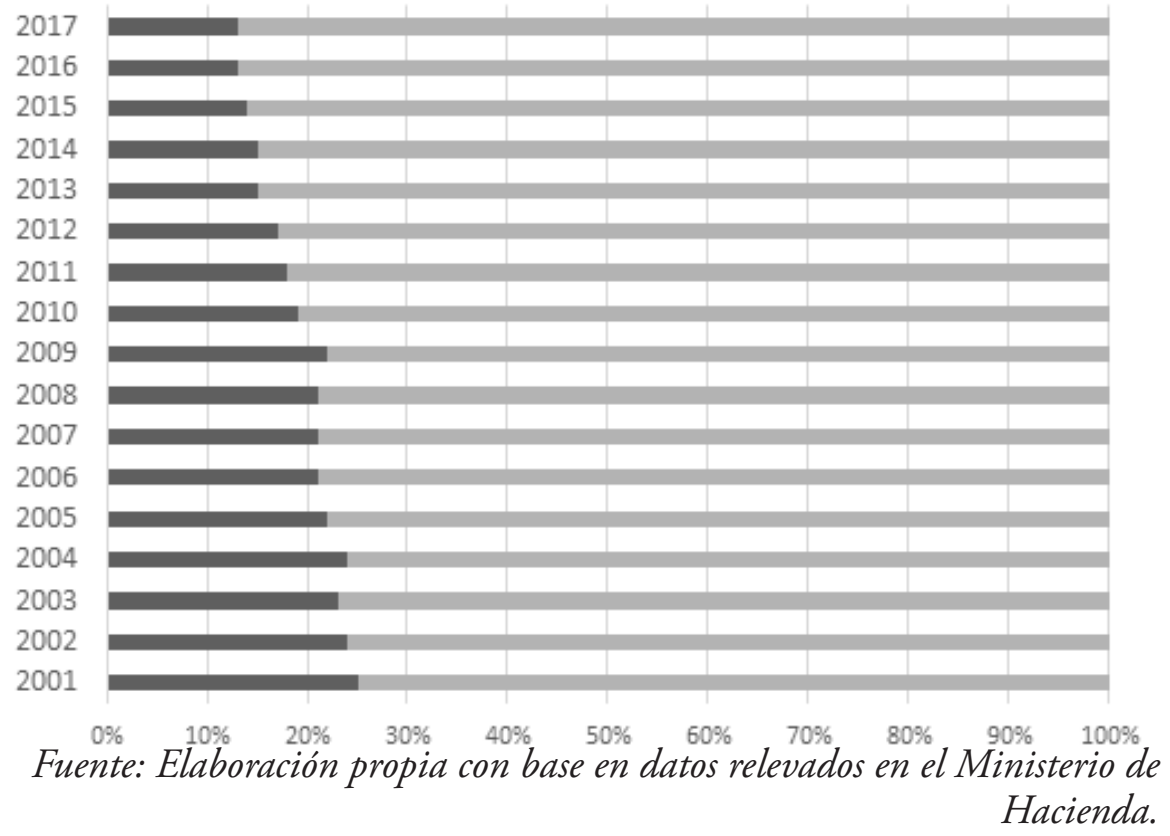


Al desagregar los datos por nivel político de conducción se observa que, en el año 2017, las mujeres representaban el 50\% en el nivel más alto de gobierno (Presidente y Vicepresidente) y solo el $10 \%$ de los ministros. Le sigue una representación femenina del $15 \%$ entre los subsecretarios y solo un $27 \%$ de mujeres en los cargos extraescalafonarios y asesores de gabinete.

Considerando el tamaño actual del sector público nacional, se observa que, en 2017, se compone de 20 ministerios y solo dos de ellos están encabezados por mujeres (el Ministerio de Desarrollo Social y, paradójicamente, el de Seguridad). Un dato clave de segregación horizontal es que, en los últimos cuatro gobiernos democráticos, el Ministerio de Desarrollo Social estuvo a cargo de una mujer. También hubo mujeres a cargo de los Ministerios de Seguridad (tres desde 2010 a 2017) y en Trabajo y Educación. De hecho, desde la década de 1980 (que coincide con vuelta a la democracia en la Argentina) hasta 2017, 15 mujeres ocuparon puestos de Ministra (y dos de ellas lo hicieron en dos oportunidades, es decir, que en esos no se abrió espacio a nuevas participantes).

En suma, en 2017, el alto gobierno se encontraba integrado por casi 900 funcionarios, dentro de los cuáles, $24 \%$ de estos puestos es ocupado por mujeres. Sin embargo, los cargos políticos de primera línea que incluyen al Presidente, Vicepresidente, ministros, secretarios y subsecretarios, estaban ocupados mayoritariamente por hombres (79\%).

Al examinar los datos referidos a la edad de los funcionarios, con base en tramos etarios de 10 ańos, encontramos un dato curioso: las mujeres que acceden al alto gobierno se encuentran en la categoría de personas mayores a 60 ańos, lo que representa un $24 \%$ del total y, dentro de la cual, las mujeres conforman el $20 \%$. En cambio, en los demás tramos etarios, encontramos siempre presencia mayoritariamente masculina: el $31 \%$ de los funcionarios tiene entre 40 y 49 ańos, de los cuales $74 \%$ son hombres. El 19\% tienen entre 20 y 39 años, también con mayorías masculinas y un $26 \%$ se encuentra en el rango etario que va desde los 50 a 59 ańos.

Respecto a la formación de estos funcionarios del alto gobierno, el $90 \%$ de ellos posee un título universitario o superior, siendo los hombres el 75\% de ellos. El 10\% del nivel educativo restante se divide en un $8 \%$ con título terciario y un $2 \%$ con título secundario.

En síntesis, si bien la participación femenina creció durante los últimos años, los hombres continúan ocupando, mayoritariamente, aquellos puestos de mayor jerarquía $y$, por ende, son quiénes mejores salarios perciben. 


\section{LA ALTA DIRECCIÓN PÚBLICA}

La ADP refiere a los cargos directivos dentro de la carrera del servicio civil que tienen la función de dirigir los procesos con los que se implementan las políticas públicas (Iacoviello y Chudnovsky 2015). En los contextos democráticos, la alternancia política implica rotación de los cargos políticos. Ese juego político puede atentar contra la necesaria estabilidad de las políticas de Estado. Además de la propia rotación democrática, hay áreas estratégicas de gobierno (como Economía) que, en el contexto de las crisis económicas recurrentes de la Argentina, suelen presentar mucha inestabilidad de los gabinetes, lo cual puede acentuar el problema. Es por eso que la alta dirección pública juega un rol estratégico.

Según la normativa, se accede a un cargo directivo mediante un sistema de concurso que evalúe la idoneidad para el cargo. El cargo dura cinco ańos porque la idea es que roten y cuando se acabe su periodo vuelvan a sus funciones anteriores dentro del servicio civil. Los cargos directivos, en orden de jerarquía, son: Nivel I (Dirección Nacional), Nivel II (Dirección de Primera Apertura), Nivel III (Dirección de Segunda Apertura) y Nivel IV (Coordinación General, Regional o Temática). Cabe aclarar que desde hace más de 10 años la mayoría de los cargos de la ADP, que deberían ser concursados de acuerdo con el SINEP, se ocupan a través de decretos de excepción al concurso y, a veces, por excepción a los requisitos para el cargo. Esto es parte del desafío de profesionalización que enfrenta masivamente la región (Chudnovsky 2017, Iacoviello y Chudnovsky 2015, Pardo 2005, Cortázar et al. 2014).

Se analizan los cargos directivos solo del escalafón SINEP, ya que es el único transversal a los organismos que integran el PEN y, además, es el más poblado (39\% del total del personal civil del PEN se concentra en ese escalafón). Dentro de SINEP, el 14\% pertenece al nivel de conducción o directivo que forma parte de la ADP y el resto pertenece al nivel administrativo.

El siguiente gráfico muestra un leve crecimiento de las mujeres en los puestos ejecutivos, tanto en 2009 como en 2017. Sin embargo, se observa una caída de la participación masculina de solo 4 puntos porcentuales en los últimos 15 años. 
Gráfico 2: Alta Dirección Pública por género

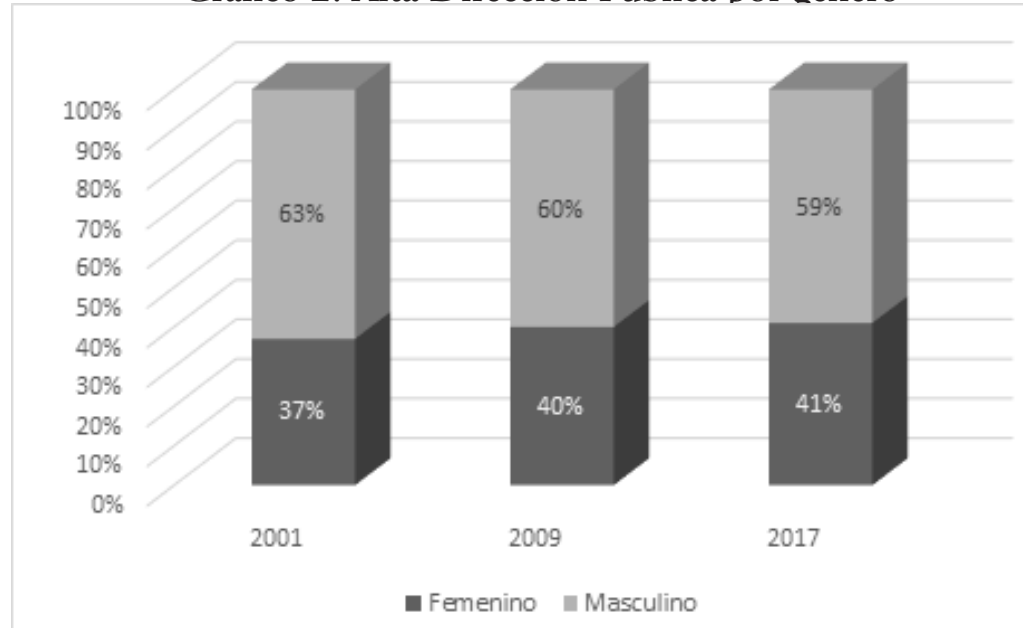

Fuente: Elaboración propia con base en datos relevados en el Ministerio de Hacienda.

La ADP reproduce, aunque en menor medida, niveles de segregación vertical. En el año 2016, las mujeres fueron titulares del $32 \%$ de las direcciones nacionales, generales y simples (contra un 38\% en 2015) y de un $33 \%$ de las coordinaciones.

Asimismo, se puede observar que en los últimos 15 años hubo una disminución de cuatro puntos porcentuales en la participación masculina, a pesar de que la cantidad de personal con estas funciones se mantuvo constante $(3 \%)$ respecto al total del personal agrupado en el escalafón SINEP (Buchner, Cafarelli y Digilio 2017).

Sin embargo, el único concurso en los últimos 8 años tampoco mostró que su presencia alterara esa tendencia. Entre los años 2010 y 2015 se llamó a una convocatoria de siete personas para ocupar cargos directivos, pertenecientes al Ministerio de Ciencia, Tecnología e Innovación Productiva. Los resultados fueron cinco cargos (71\%) designados para los hombres y dos (29\%) para las mujeres. Esos dos puestos ocupados por mujeres son los dos más bajos de la jerarquía, en comparación con los otros cinco.

En síntesis, los datos confirman que el nivel de representación femenina en puestos de decisión del PEN es bajo. 


\section{Diferencias SALARIALES EN LA ADP}

$\mathrm{Al}$ analizar los salarios de la $\mathrm{ADP}$, encontramos que los hombres perciben salarios más altos que las mujeres. Si observamos los sueldos, con base en tramos de salarios cada $\$ 30.000$ (pesos argentinos), vemos que el $71 \%$ de los hombres con cargos directivos se encuentra en el más alto, mientras que el $67 \%$ de las mujeres se concentra en el tramo salarial más bajo.

Tabla 2: Distribución de la ADP por tramos salariales y género, 2017 Tramo de ingreso Femenino Masculino

\begin{tabular}{ccc}
\hline Hasta $\$ 30.000$ & $67 \%$ & $33 \%$ \\
$\$ 31.000$ a $\$ 60.000$ & $45 \%$ & $55 \%$ \\
$\$ 61.000$ a $\$ 90.000$ & $39 \%$ & $61 \%$ \\
$\$ 91.000$ a $\$ 120.000$ & $33 \%$ & $67 \%$ \\
Mayor a $\$ 120.000$ & $29 \%$ & $71 \%$ \\
Fuente: Elaboración propia con base en datos de Buchner, Cafarelli y Digilio
\end{tabular}
(2017).

El 59\% de los cargos directivos están ocupados por hombres y el $41 \%$ por mujeres y la mayoría de los hombres se encuentra en los tramos salariales superiores (más del 60\%). Es decir, incluso compartiendo el segmento directivo, los hombres obtienen una mejor paga que las mujeres.

Esto es consistente con resultados del último informe de Distribución del Ingreso presentado por el Instituto Nacional de Estadísticas y Censos (INDEC 2017) que señala que, si bien el mercado laboral argentino se compone casi de la misma cantidad de hombres y mujeres que reciben ingresos, las mujeres perciben un $27 \%$ menos y son mayoría en el tramo de menores ingresos. En el decil más alto de ingresos, el 6,3\% son hombres y 3,7\% son mujeres (Buchner, Cafarelli y Digilio 2017).

En cuanto a las características de la ADP, analizamos la cantidad de hombres y mujeres por tramos de edad, en categorías de 10 años. 


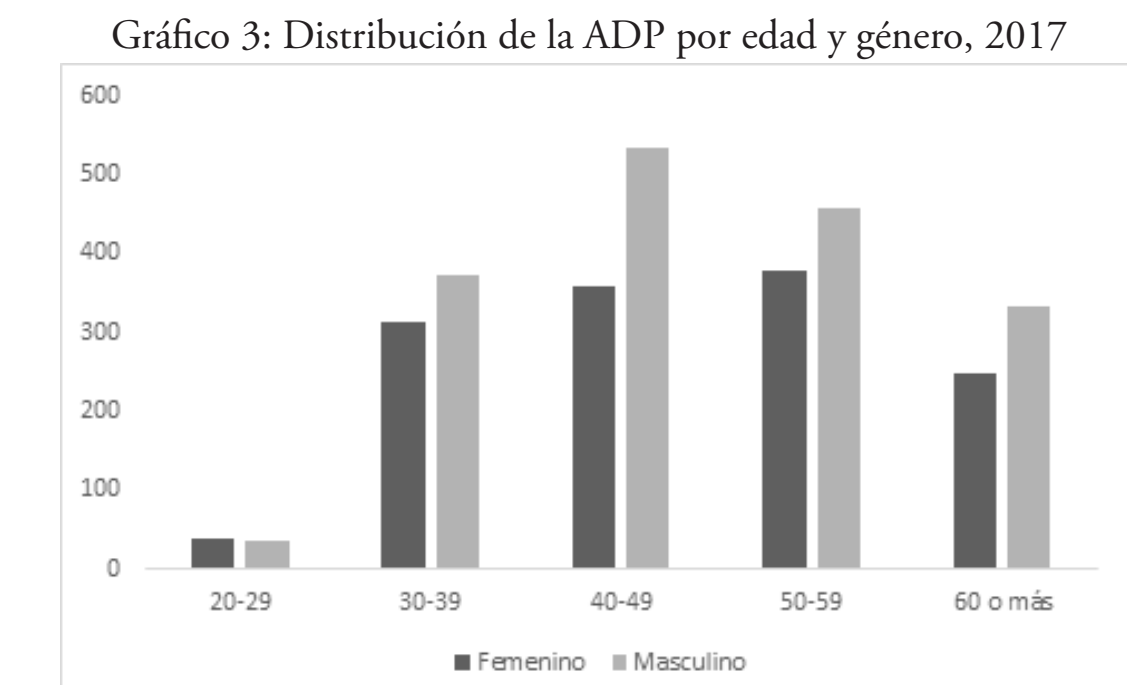

Fuente: Elaboración propia con base en datos del Ministerio de Hacienda de la Nación.

El Gráfico 3 muestra que la mayoría de las mujeres en cargos directivos tienen entre 50 y 59 años, mientras que la mayoría de los hombres se encuentra en un intervalo etario de menor edad: entre 40 a 49 años.

En cuanto al nivel educativo, se observa que el $88 \%$ de los directores públicos poseen un título universitario, un $9 \%$ poseen uno secundario, y el resto tiene títulos terciarios (3\%). De ese total de universitarios, los hombres representan el $57 \%$ y las mujeres el resto (43\%). Si bien es requisito para acceder al cargo directivo poseer un título universitario, como mencionamos anteriormente, existe la posibilidad de designar personas que no cumplan con los requisitos mínimos de acceso al puesto, mediante la excepción.

Sigue habiendo mayoría de hombres al analizar los puestos de ADP con títulos terciarios y secundarios, con $67 \%$ y $72 \%$, respectivamente. En resumen, es importante destacar que, si bien para acceder a la ADP se requieren ciertos requisitos mínimos relacionados al nivel académico del agente y a la antigüedad laboral, estos pueden estar exceptuados al momento de la designación realizada con base en criterios de confianza (no mérito). De aquí resulta que, al analizar la distribución tanto por nivel educativo o tramos de edad, el resultado muestre hombres sin título universitario o muy jóvenes (de 20 a 29 años) en cargos directivos. 


\section{NiVEl ADMINISTRATIVO}

El siguiente gráfico presenta la cantidad de personas incluidas en el escalafón SINEP para el nivel administrativo (ni políticos, ni directivos) examinado por género para los años 2001 a 2017.

\section{Gráfico 4: Administrativos por género, 2001-2017}

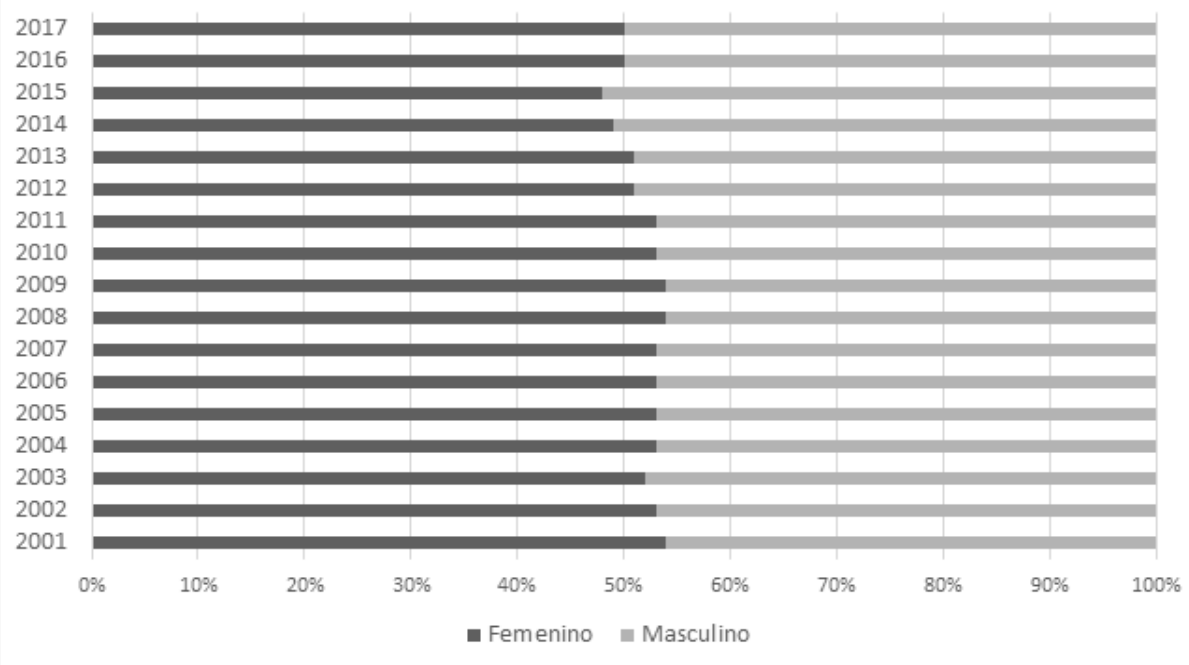

Fuente: Elaboración propia con base en datos relevados en el Ministerio de Hacienda.

Considerando lo presentando en las secciones anteriores, cabe destacar que, aunque en la actualidad, las mujeres representan el 54\% de la fuerza de trabajo empleada por la administración central del PEN, esta composición casi paritaria en la base piramidal, no se refleja en las altas esferas o puestos de decisión tanto del alto gobierno como de la ADP. La presencia femenina disminuye a medida que se asciende en la pirámide de poder de la administración pública nacional, tal como se expuso en la Tabla 1.

Ahora bien, al desagregar los datos de entre aquellos que revisten en planta permanente y aquellos que tienen un contrato, los resultados muestran una distribución equitativa de hombres y mujeres en este nivel. La siguiente tabla muestra la evolución de estos datos para los años 2003 a 2017. 
Tabla 3: Administrativos por modalidad de contratación, 2003-2017 Planta Permanente Contratos

Total

\begin{tabular}{ccccccc}
\hline Año & Femenino & Masculino & Femenino & Masculino & Femenino & Masculino \\
2003 & $50 \%$ & $50 \%$ & $21 \%$ & $79 \%$ & $48 \%$ & $52 \%$ \\
2005 & $50 \%$ & $50 \%$ & $51 \%$ & $49 \%$ & $51 \%$ & $49 \%$ \\
2007 & $53 \%$ & $47 \%$ & $54 \%$ & $46 \%$ & $53 \%$ & $47 \%$ \\
2009 & $53 \%$ & $47 \%$ & $54 \%$ & $46 \%$ & $54 \%$ & $46 \%$ \\
2011 & $52 \%$ & $48 \%$ & $54 \%$ & $46 \%$ & $53 \%$ & $47 \%$ \\
2013 & $52 \%$ & $48 \%$ & $53 \%$ & $47 \%$ & $53 \%$ & $47 \%$ \\
2015 & $52 \%$ & $48 \%$ & $52 \%$ & $48 \%$ & $52 \%$ & $48 \%$ \\
2017 & $52 \%$ & $48 \%$ & $54 \%$ & $46 \%$ & $54 \%$ & $46 \%$
\end{tabular}

Fuente: Elaboración propia con base en datos relevados en el Ministerio de Hacienda.

De la tabla anterior, se pueden ver los porcentajes que pertenecen a la cantidad de mujeres y hombres contratados, donde se observa, por un lado, una disparidad importante los primeros años: $21 \%$ mujeres y $79 \%$ hombres y, por el contrario, cantidad de contratos por género equitativos a partir del año 2005 en adelante. Por otro lado, el personal de planta permanente muestra una distribución por género más lineal, teniendo las mujeres, en promedio, una participación del 52\% durante el período considerado.

\section{LA PROMOCIÓN DE JERARQUÍA}

Para ascender en el servicio civil, existe una combinación de progreso horizontal con base en el desempeño y la acreditación de competencias laborales o actividades de capacitación con una promoción vertical a través de mecanismos de selección por llamado a concurso.

Los servidores públicos acceden a diferentes niveles dentro de la jerarquía del sector público argentino (siendo el nivel $\mathrm{A}$ el más alto y $\mathrm{F}$ el más bajo). A su vez, promocionan horizontalmente mediante grados que se obtienen por paso del tiempo con capacitación y evaluaciones positivas de desempeño, de carácter anual. Es decir, el nivel A con grado 10 o mayor sería la jerarquía más alta dentro de este universo.

En el año 2017, los servidores públicos que se encuentra en los niveles más altos del escalafón (nivel A) eran mayoritariamente hombres (54\%). Las mujeres ocupan mayoritariamente los siguientes niveles: $58 \%$ en los niveles $\mathrm{B}$ y $\mathrm{C}$, mientras que el $51 \%$ en el D. En tanto, los niveles $\mathrm{E}$ y 
F están ocupados mayoritariamente por hombres, con el 54\% y 59\%, respectivamente. Esos dos niveles son los no profesionales.

La siguiente tabla resume toda la información referida al nivel escalafonario de los administrativos dentro del escalafón SINEP para el año 2017.

Tabla 4: Administrativos por nivel escalafonario. 2017

\begin{tabular}{ccccccccc} 
& & A & B & C & D & E & F & Total \\
\hline Planta permanente & F & $40 \%$ & $50 \%$ & $58 \%$ & $55 \%$ & $47 \%$ & $30 \%$ & $52 \%$ \\
& M & $60 \%$ & $50 \%$ & $42 \%$ & $45 \%$ & $53 \%$ & $70 \%$ & $48 \%$ \\
Contratos & F & $50 \%$ & $60 \%$ & $58 \%$ & $49 \%$ & $45 \%$ & $46 \%$ & $54 \%$ \\
& M & $50 \%$ & $40 \%$ & $42 \%$ & $51 \%$ & $55 \%$ & $54 \%$ & $46 \%$ \\
Total & F & $46 \%$ & $58 \%$ & $58 \%$ & $51 \%$ & $46 \%$ & $41 \%$ & $54 \%$ \\
& M & $54 \%$ & $42 \%$ & $42 \%$ & $49 \%$ & $54 \%$ & $59 \%$ & $46 \%$
\end{tabular}

Fuente: Elaboración propia con base en datos relevados en el Ministerio de Hacienda.

Al desagregar los datos por modalidad de empleo, en la planta permanente vemos una tendencia parecida, donde el nivel más alto (A) tiene mayoría masculina. En tanto, el siguiente nivel (B) se encuentra equilibrado, siguiendo los niveles $\mathrm{C}$ y $\mathrm{D}$ con el 58\% y 55\% de mayorías femeninas. Luego, los niveles más bajos presentan mayor cantidad de hombres. Para el caso del personal contratado, el nivel más alto tiene cantidades equitativas de hombres y mujeres (50\%), los niveles $\mathrm{B}$ y $\mathrm{C}$ son contratos mayoritariamente de mujeres, mientras que los más bajos $(\mathrm{D}, \mathrm{E}$ y F) son contratos con mayorías masculinas.

Del análisis de la cantidad de personas por nivel educativo, encontramos que el nivel más poblado en este segmento es el secundario.

$\mathrm{Al}$ analizarlos por género, las mujeres tienen mayoría de títulos terciarios (56\%) y universitarios (52\%). El nivel secundario presenta cantidades equitativas de hombres y mujeres, y finalmente el nivel primario tiene más cantidad de hombres (58\%).

El siguiente gráfico muestra la distribución porcentual de este personal por nivel educativo y género. 
Gráfico 5: Educación por género de administrativos, 2017

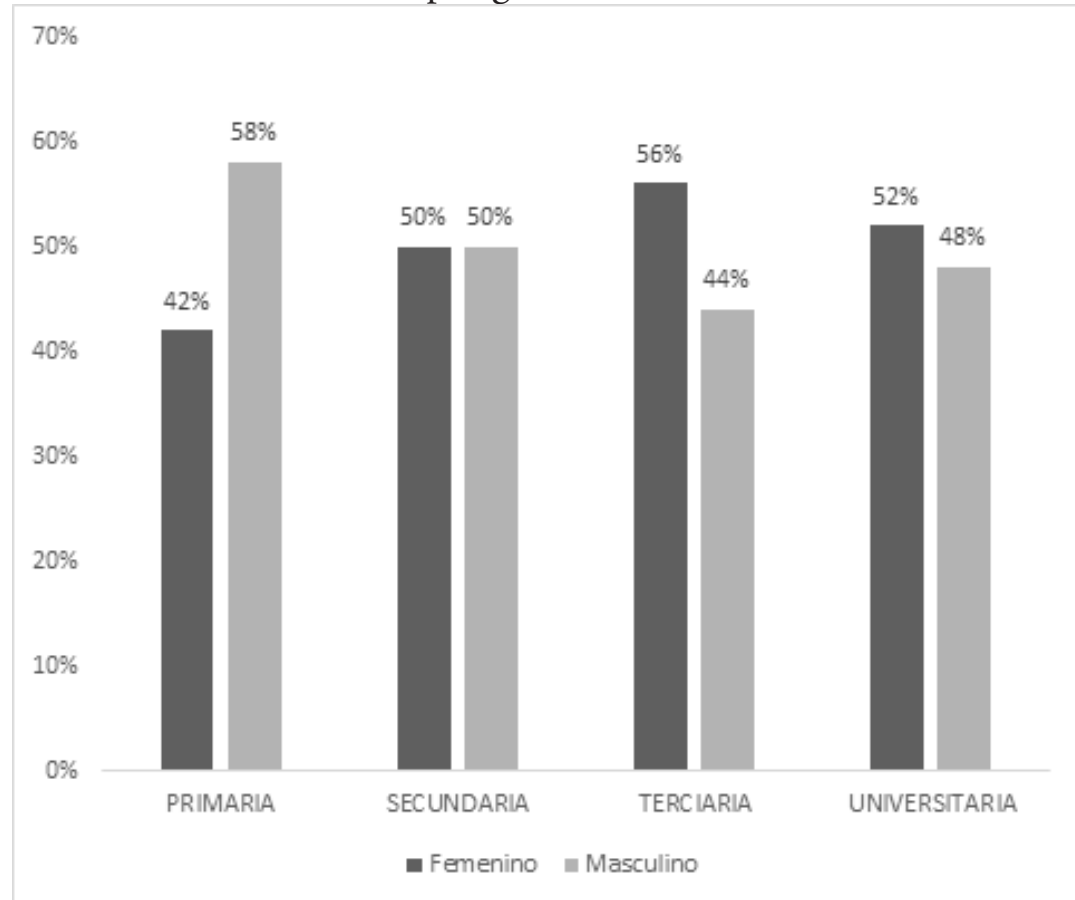

Fuente: Elaboración propia con base en datos relevados en el Ministerio de Hacienda.

$\mathrm{Al}$ analizar la cantidad de cargos por rango etario, considerando también intervalos cada 10 años, el 55\% de mujeres tiene entre 30 y 39 ańos. El $25 \%$ de los servidores públicos tiene entre 40 y 49 ańos y $55 \%$ de ellos son mujeres. Solamente un $14 \%$ tiene entre 20 y 29 años, un $18 \%$ entre 50 y 59 ańos y el resto (11\%) tiene más de 60 años.

\section{CONCLUSIONES}

La evidencia presentada muestra que la administración pública nacional argentina presenta segregación de género: los hallazgos más significativos son que, en el año 2017, los hombres ocupaban la mayoría de las posiciones del gabinete político y de las posiciones gerenciales dentro del servicio civil. En nivel de la burocracia adminnistrativa, la presencia de hombres y mujeres tiende a ser más númericamente balanceada, pero son más las mujeres con títulos universitarios y, a pesar de ello, se concentran en los niveles más bajos de la jerarquía y ganan menos que los hombres en la misma posición laboral. Asimismo, se concentran en las áreas más feminizadas como, por ejemplo, el Ministerio de Desarrollo Social. Estos hallazgos son consistentes con el hecho de que, en América Latina y en 
la mayoría de las esferas de la actividad del sector público, las mujeres permanecen seriamente subrepresentadas en posiciones de autoridad política y administrativa.

Este trabajo se centró en diagnosticar qué sucede en el Poder Ejecutivo argentino, como caso exploratorio para esbozar posibles hipótesis de este fenómeno que luego se puedan comprobar en un estudio de más casos. Encontramos un severo contraste entre el avance en el Poder Legislativo y el Ejecutivo: en este último, existe presencia de mujeres si uno observa lo datos agregados. Sin embargo, si estos se desagregan a la luz de lo que la literatura señala, se encuentra que existe segregación, tanto vertical como horizontal y una fuerte presencia de techo de cristal. ¿Qué explica la diferencia en un caso icónico en materia de acción afirmativa, como el caso argentino? ¿Por qué la administración pública muestra segregación de género tanto vertical como horizontal? Una burocracia representativa implica reflejar la composición de la sociedad en la que opera. Estos resultados la presencia de más mujeres en el poder (legislativo) no necesariamente mejoran la situación de todas las mujeres en las organizaciones. La literatura sobre burocracias pasivas y activas puede arrojar luz sobre esta paradoja: la presencia de mujeres en la legislatura puede mejorar la situación de las mujeres en general (sociedad) pero no necesariamente para dentro (organización). Y la mejora de la representación activa dentro de una organización que refleja la diversidad de la sociedad, no necesariamente se traduce en una presentación más política. Contribuimos al debate académico ya que la mayor parte de la investigación se ha centrado en el vínculo entre la representación pasiva y la representación activa orientada hacia la sociedad. En cambio, nuestro objetivo es explorar lo que sucede dentro de un mismo Estado federal. 


\section{REFERENCIAS}

Acker, J. R. (1980). Women and stratification: A review of recent literature. Contemporary Sociology, 9 (1), 25-35.

. (1990). Hierarchies, jobs, bodies: A theory of gendered organizations. Gender \& Society, 4 (2), 139-158.

. (1998). The future of 'Gender and organizations': Connections and boundaries. Gender, Work \& Organization, 5 (4), 195-206.

Alkadry, M. G. y Tower, L. E. (2014). Women and public service: Barriers, challenges and opportunities. Nueva York: Routledge.

Alkadry, M. G., Bishu, Sebawit G. y Sussanah Bruns, A. (2019). Beyond Representation: Gender, authority, and city managers. Review of Public Personnel Administration, 39 (2), 300-319.

Andrews, R. y Johnston Miller, K. (2013). Representative bureaucracy, gender, and policing: The case of domestic violence arrests in England. Public Administration, 91 (4), 998-1014.

Atkins, D. N. y Wilkins, V. M. (2013). Going beyond reading, writing, and arithmetic: The effects of teacher representation on teen pregnancy rates. Journal of Public Administration Research and Theory, Vol. 23 (4), 771-790.

Bacchi, C. (2006). Arguing for and against quotas: theoretical issues”. En Dahlerup, D. (Ed.), Women, quotas and politics. Nueva York: Routledge.

Barreiro, L., López, O., Soto, C. y Soto, L. (2004). Sistemas electorales y representación femenina en América Latina. Santiago: CEPAL.

Bishu, S. y Headley, A. M. (2020). Equal employment opportunity: Women bureaucrats in male-dominated professions. Public Administration Review, online first, 1-12.

Bishu, S. G. y Alkadry, M. (2017). A systematic review of the gender pay gap and factors that predict it. Administration \& Society, 49 (1), 65104. 
Bowling, C. J., Kelleher, C. A., Jones, J. y Wright, D. S. (2006). Cracked ceilings, firmer floors, and weakening walls: Trends and patterns in gender representation among executives leading American state agencies, 1970-2000. Public Administration Review, 66 (6), 823-836.

Bradbury, M. y Kellough, J. E. (2011). Representative bureaucracy: Assessing the evidence on active representation. The American Review of Public Administration, 41 (2), pp.157-167.

Buchner, L., Cafarelli, M. L. y Digilio, G. T. (2017). La participación de la mujer en la alta dirección pública: los desafíos en la inclusión en la experiencia argentina. Ponencia presentada en el XXII Congreso Internacional del CLAD sobre la Reforma del Estado y de la Administración Pública, 14 al 17 de noviembre, Madrid, España.

Bullard, A. M. y Wright, D. S. (1993). Circumventing the glass ceiling: Women Executives in American state governments. Public Administration Review, 53 (3), 189-202.

Camarena, M. E. y Saavedra, M. L. (2018). El techo de cristal en México. Revista La Ventana, 5 (47), 312-347.

Cáceres-Rodríguez, R. (2013). The glass ceiling revisited: Moving beyond discrimination in the study of gender in public organizations. Administration \& Society, 45 (6), 674-709.

Calvo, E. y Murillo, M. V. (2004). Who delivers? Partisan clients in the Argentine electoral market. American Journal of Political Science, 48 (4), 742-757.

Chappell, L. (2013). The state and governance. En Waylen, G., Celis, K., Kantola, J. y Weldon, S. L. (Eds.), The Oxford handbook of gender and politics. Oxford y Nueva York: Oxford University Press.

Choi, S. (2018). Pay equity in government: Analyzing determinants of gender pay disparity in the US federal government. Administration \& Society, 50 (3), 346-371.

Chudnovsky, M. (2017). La tensión entre mérito y confianza en la alta dirección pública de América Latina. Revista del CLAD. Reforma y Democracia, 69, 5-40. 
Chudnovsky, M. y Caffarelli, M. L. (2018). Los cambios en las estructuras organizacionales del Estado y su vínculo con la composición del empleo público. Argentina, 2003-2016. Foro Internacional, 58 (2), 275-312.

Centro de Implementación de Políticas Públicas para la Equidad y el Crecimiento (CIPPEC) (2017). GPS del Estado: Cuánto y cómo cambió el sector público nacional. Buenos Aires: CIPPEC.

Connell, R. (2006). Glass ceilings or Gendered Institutions? Mapping the gender regimes of public sector worksites. Public Administration Review, 66 (6), 837-849.

Cortázar Velarde, J. C., Lafuente, M. y Sanginés, M. (Eds.) (2014). Una década de reformas del servicio civil en América Latina (2004-2013). Washington: Banco Interamericano de Desarrollo.

D'Agostino, M. J. (2015). The difference that women make: Government performance and women-led agencies. Administration \& Society, 47 (5), 532-548.

Dador Tozzini, M. J. (2007). Contexto político de la región: desafíos y oportunidades para las políticas de género. En Falú, A. y Segovia, O. (Eds.), Ciudades para convivir sin violencia hacia las mujeres. Debate para la construcción de propuestas. Santiago: Ediciones SUR.

Daly, M. (2005). Gender mainstreaming in theory and practice. Social Politics: International Studies in Gender, State \& Society, 12 (3), 433450 .

Dirección de Evaluación Presupuestaria Nacional (2017). Poder Ejecutivo Nacional, empresas y sociedades del Estado, otros entes del sector público nacional no financiero. Octubre de 2017. Disponible en http://www2.mecon.gov.ar/hacienda/dnoyssp/informe/201710.pdf [02-06-2020].

Dolan, J. (2004). Gender equity: Illusion or reality for women in the federal executive. Public Administration Review, 64 (3), 299-308.

Echabarría, Koldo (Ed.) (2006). Informe sobre la situación del servicio civil en América Latina. Washington: Banco Interamericano de Desarrollo. 
Fernández, S., Malatesta, D. y Smith, C. R. (2013). Race, gender, and government contracting: Different explanations or new prospects for theory? Public Administration Review, 73 (1), 109-120.

Gaete, R. y Álvarez, J. (2020). Alta dirección pública y techo de cristal. Acceso de las mujeres a los puestos directivos en Chile. Espiral. Estudios sobre Estado y Sociedad, XXVII (77), 179-222.

Gaete, R., Álvarez, J. y Ramírez, M. (2019). Reflexiones y experiencias de profesoras-investigadoras mexicanas sobre el techo de cristal. Calidad en la Educación, 50, 457-491.

García Prince, E. (2003). Hacia la institucionalización del enfoque de género en políticas públicas. Caracas: ILDIS.

Grindle, M. (2012). Jobs for the boys: Patronage and the state in comparative perspective. Cambridge: Harvard University Press.

Iacoviello, M. y Chudnovsky, M, (2015). La importancia del servicio civil en el desarrollo de capacidades estatales en América Latina. Washington: Banco de Desarrollo de América Latina.

Iacoviello, M., Llano, M. y Strazza, L. (2012). Fortalecimiento de la función directiva pública: Exploración a partir de una selección de experiencias latinoamericanas y anglosajonas. Revista del CLAD. Reforma y Democracia, 50, 45-82.

Iacoviello, M. y Pulido, N. (2012). Representatividad, participación y política: nuevo foco para el viejo debate sobre la función pública en América Latina. Buen Gobierno, 12, 95-114.

Iacoviello, M., Rodríguez Gustá, A. L. y Zuvanic, L. (2010). The weakest link: The bureaucracy and civil service systems in Latin America. Scartascini, C., Stein, E. y Tommasi, M. (Eds.), How democracy works. Political institutions, actors, and arenas in Latin American policymaking. Washington y Cambridge: Inter-American Development Bank, David Rockefeller Center for Latin American Studies, Harvard University.

Instituto Nacional de Estadísticas y Censos (INDEC) (2017). Evolución de la distribución del ingreso. Primer semestre de 2017 Buenos Aires: INDEC. 
Jin, J. (2017). Female participation and corruption in the public sector. International Review of Public Administration, 22 (4), 305-319.

Keiser, L., Wilkins, V., Meier, Kenneth J. y Holland, C. (2002). Lipstick and logarithms: Gender, institutional context, and representative bureaucracy. American Political Science Review, 96 (3), 553-564.

Kennedy, B. (2014). Unravelling representative bureaucracy: A systematic analysis of literature. Administration \& Society, 46 (4), 395-421.

Kerr, B., Miller, W. y Reid, M. (2002). Sex-based occupational segregation in US state bureaucracies, 1987-97. Public Administration Review, 62 (4), 412-423.

Kingsley, J. D. (1944). Representative bureaucracy: An interpretation of the British civil service. Yellow Springs: Antioch Press.

Kranz, H. (1976). The participatory bureaucracy: Women and minorities in a more representative public service. Lexington: Lexington Books.

Lewis, G. B. y Pitts, D. W. (2011). Representation of lesbians and gay men in federal, state, and local bureaucracies. Journal of Public Administration Research and Theory, 21 (1), 159-180.

Lewis, G. B. y Soo Oh, S. (2009). A Major difference? Fields of study and male-female pay differences in federal employment. The American Review of Public Administration, 39 (2), 107-124.

Marx, J., Borner, J. y Caminotti, M. (2007). Las legisladoras: Cupos de género y politica en Argentina y Brasil. Buenos Aires: Siglo XXI.

Meier, K. J. (1993). Representative bureaucracy: A theoretical and empirical exposition. En Perry, J. (Ed.), Research in public administration. Greenwich: JAI Press.

Meier, K. J. y Nicholson-Crotty, J. (2006). Gender, representative bureaucracy, and law enforcement: The case of sexual assault. Public Administration Review, 66 (6), 850-860.

Meier, Kenneth J. y Nigro, Lloyd G. (1976). Representative bureaucracy and policy preferences: A study in the attitudes of federal executives. Public Administration Review, 36 (4), 458-469. 
Meier, K. J. y Stewart, J. (1992). The impact of representative bureaucracies: Educational systems and public policies. The American Review of Public Administration, 22 (3), 157-171.

Organización de las Nacinoes Unidas (ONU) (2017). Las Naciones Unidas y la mujer. Disponible en http://www.un.org/es/sections/issues-depth/ women/index.html [01-06-2020].

Opstrup, N. y Villadsen, A. R. (2014). The right mix? Gender diversity in top management teams and financial performance. Public Administration Review, 75 (2), 291-301.

Pardo, M. C. (2005). El servicio profesional de carrera en México: De la tradición al cambio. Foro Internacional, 45 (4), 599-634

Park, S. (2013). Does gender matter? The effect of gender representation of public bureaucracy on governmental performance. The American Review of Public Administration, 43 (2), 221-242.

Rhodebeck, L. A. (1996). The structure of men's and women's feminist orientations: Feminist identity and feminist opinion. Gender \& Society, 10 (4), 386-403.

Riccucci, N. M. y Saidel, J. R. (1997). The representativeness of state-level bureaucratic leaders: A missing piece of the representative bureaucracy puzzle. Public Administration Review, 57 (5), 423-430.

. (2001). The demographics of gubernatorial Appointees: Toward an explanation of variation. Policy Studies Journal, 29 (1), 1122.

Ridgeway, C. L. y Correll, S. J. (2004). Unpacking the gendered system: A theoretical perspective on gender beliefs and social relation. Gender \& Society, 18 (4), 510-531.

Rodríguez Gustá, A. L. (2008). Las políticas sensibles al género: variedades conceptuales y desafíos de intervención. Temas y debates: Revista Universitaria de Ciencias Sociales, 16, 109-130.

Rodríguez Gustá, A. L. y Caminotti, M. (2010). Políticas públicas de equidad de género: las estrategias fragmentarias de la Argentina y Chile. Revista SAAP: Sociedad Argentina de Análisis Politico, 4 (1), 85-110. 
Ryan, M. K. y Haslam, A. (2007) The glass cliff: Exploring the dynamics surrounding the appointment of women to precarious leadership positions. The Academy of Management Review, 32 (2), 549-572.

Sabharwal, M. (2013). From glass ceiling to glass cliff: Women in senior executive service. Journal of Public Administration Research and Theory, 25 (2), 399-426.

Selden, S. C. (1997). Representative bureaucracy: Examining the linkage between passive and active representation in the Farmers Home Administration. The American Review of Public Administration, 27 (1), $22-42$.

- (2006). A Solution in search of a problem? Discrimination, affirmative action, and the new public service. Public Administration Review, 66 (6), 911-923.

Stivers, C. (2000). Bureau men, settlement women. Constructing public administration in the progressive era. Lawrence: University Press of Kansas.

. (2002). Gender images in public administration: Legitimacy and the administrative state. Thousand Oaks: Sage.

Wilkins, V. M. (2007). Exploring the causal story: Gender, active representation, and bureaucratic priorities. Journal of Public Administration Research and Theory, 17 (1), 77-94.

World Economic Forum (2017). The Global Gender Gap Report 2017. Ginebra: World Economic Forum.

World Economic Forum.

. (2018). The Global Gender Gap Report 2018. Ginebra:

Recibido: 11-02-2020

Aceptación de la versión final: 01-06-2020 